\title{
POPULAÇÃO DE NEMATOIDES EM RELAÇÃO À ESPESSURA DA COBERTURA VEGETAL NO CULTIVO DA ALFACE
}

Bruna Coelho de Lima, Heloisa Maria da Cruz Jardim, Rita de Cássia Lima Mazzuchelli; Fabio Fernando de Araujo

Universidade do Oeste Paulista - UNOESTE, Presidente Prudente, SP. E-mail: brunacoelhoo@outlook.com

\section{RESUMO}

O objetivo do presente trabalho foi avaliar a população de nematoides no solo no cultivo da alface submetida a diferentes resíduos e níveis de cobertura do solo. O experimento foi realizado em Álvares Machado no sitio São Lourenço. Foram avaliados dois tipos de resíduos como casca de amendoim e cana de açúcar triturada e também dois níveis de cobertura do solo de $3 \mathrm{e} 6 \mathrm{~cm}$ em cultivos sucessivos em diferentes épocas do ano. O estudo foi conduzido em canteiros de horta comercial, atendendo ao manejo de condução das plantas adotado pelos produtores. Trinta dias e trinta e cinco dias no primeiro e segundo ciclo respectivamente, após o plantio foram coletadas amostra de solo para análises de nematoides no solo. A cobertura morta composta pelo bagaço de cana-de-açúcar ganhou destaque referente ao aumento populacional de nematoides do gênero Meloidogyne. A camada com espessura de $3 \mathrm{~cm}$ de resíduo de cana-de-açúcar foi suficiente para elevar a população de nematoides no solo.

Palavras-chave: Meloidogyne, Pratylenchus, hortaliça, bagaço-de-cana, casca de amendoim.

\section{POPULATION OF NEMATOIDES IN RELATION TO THICKNESS OF VEGETABLE COVERAGE IN ALFACE CULTIVATION}

\section{ABSTRACT}

The objective of the present work was to evaluate the soil nematode population in lettuce cultivation submitted to different residues and levels of soil cover. The experiment was carried out in Álvares Machado at the São Lourenço site. Two types of residues such as peanut shell and crushed sugar cane were evaluated, as well as two levels of soil cover of 3 and $6 \mathrm{~cm}$ in successive crops at different times of the year. The study was conducted in commercial vegetable garden beds, taking into account the management of the plants adopted by the producers. Thirty days and thirty-five days in the first and second cycle respectively, after planting soil samples were collected for nematode analyzes in the soil. The mulch made up of sugarcane bagasse was highlighted by the population increase of nematodes of the genus Meloidogyne. The $3 \mathrm{~cm}$ thick layer of sugarcane residue was enough to raise the nematode population in the soil.

Key words: Meloidogyne, Pratylenchus, greenery, sugarcane bagasse, peanut bark.

\section{INTRODUÇÃO}

A alface (Lactuca sativa L.) é considerada a hortaliça folhosa de maior distribuição, sendo atualmente, cultivada em vários países. Seu cultivo é de modo intenso e comumente exercido pela agricultura familiar, a qual é responsável pela geração de empregos (ALENCAR et al, 2012).O emprego da cobertura morta no cultivo da alface tem demonstrado ser uma prática determinante, 
quando se busca um aumento na qualidade da produção, no entanto, para se tornar viável ao produtor, é preciso partir em busca de outros tipos de cobertura, preferindo aquelas que encontram - se disponíveis na região de cultivo (SANTOS, 2011).

Entre as espécies usadas para a cobertura morta de solo, sobressaem - se integrantes das famílias das leguminosas e das gramíneas. As leguminosas, pelo fato da fixação de nitrogênio através da simbiose com bactérias, proporcionando adição desse elemento no sistema de produção (PADOVAN et al., 2002), além do mais, sua palhada fragmentada e posta no solo, possui uma rápida decomposição e liberação de nutrientes favorecendo a nutrição da planta (AITA; GIACOMINI, 2003). Em contrapartida, as gramíneas geralmente, dispõem de uma decomposição mais vagarosa, podendo ainda, provocar a imobilização de nutrientes no solo (ESPINDOLA et al., 2006).

No Brasil, mesmo com os estudos em relação ao reaproveitamento de biomassa dos resíduos agroindustriais, essa matéria é pouco aproveitada, sendo desprezada e entregue a decomposição natural, gerando produtos nocivos ao ambiente como gás carbônico e metano (DIAS et al, 2012). De acordo com Lima et al. (2010), o aproveitamento desses resíduos propõe a auto sustentabilidade da propriedade rural e além do mais, auxilia na reposição de nutrientes extraídos pelas plantas ao longo de seu ciclo e beneficia as características físicas, químicas e biológicas do solo, transformando em um sistema sustentável.

O objetivo do presente trabalho foi avaliar a população de nematoides no solo no cultivo da alface submetida a diferentes resíduos e níveis de cobertura do solo.

\section{METODOLOGIA}

O experimento foi realizado no período de dezembro de 2016 a maio de 2017, em horta comercial localizado no município de Álvares Machado, São Paulo, no sítio São Lourenço. O clima da região é considerado subtropical tendo uma temperatura média de 23 ํ C e uma precipitação de $1244 \mathrm{~mm}$, onde o verão é bem quente e inverno com temperatura moderada e pouca chuva.

O local de desenvolvimento do experimento já era cultivado com hortaliças em canteiros por vários anos. Neste local foram reservados canteiros para realização do experimento. Foram realizadas análises prévias de fertilidade do solo.

Foram utilizados dois tipos de resíduos no experimento: casca de amendoim e bagaço de cana. Os resíduos foram dispostos nos canteiros visando formação de dois níveis de altura do resíduo em relação ao solo: 3 e $6 \mathrm{~cm}$. Desta forma o delineamento experimental ficou definido como um fatorial $2 \times 2+1$, com quatro repetições. Onde o tratamento +1 será o cultivo no solo sem nenhuma cobertura. As parcelas (resíduos) e subparcelas (níveis) foram demarcadas dentro dos canteiros com 20 metros de comprimento por um metro de largura. Nesses canteiros foram definidas as parcelas de 10 metros de comprimentos e as subparcelas de 5 metros de comprimento. Com a adubação de plantio foi de $500 \mathrm{~kg} \mathrm{ha}^{-1}$.

Cultivou-se a alface (Lactuca sativa), cultivar Vanda. A produção das mudas, transplantes e condução da cultura foram realizadas de acordo com as recomendações técnicas regionais. $A$ área experimental era utilizada a irrigação por aspersão convencional.

Decorridos 35 e 30 dias do primeiro e cultivo do segundo ciclo respectivamente, foram coletadas amostras de solo para realização de análises nematológicas, seguindo metodologia de Jenkins (1964). 
Para análise estatística utilizou-se o programa Assistat. Para análise de variância foi utilizado o teste de Dunnet e Tukey a 5\% de probabilidade.

\section{RESULTADOS}

A avaliação da população de nematoides dos gêneros Meloidogyne, Pratylenchus e número de ovos no primeiro ciclo produtivo, apresentou que os tratamentos não diferiram em relação ao tratamento controle, com o solo descoberto. Também não houveram diferenças significativas quando comparadas as espécies de coberturas do solo. Entretanto, quando comparadas os níveis de resíduos do solo foram constatadas que a maior cobertura do solo, $6 \mathrm{~cm}$ dos resíduos proporcionaram aumento na população de Meloidogyne e no número de ovos, verificado na tabela 1.

Tabela 1 - População de nematoides no solo na produção no primeiro cultivo de alface sobre diferentes resíduos, em diferentes alturas.

\begin{tabular}{|c|c|c|c|}
\hline Fatorial $\mathrm{x}$ testemunha & Meloidogyne & Pratylenchus & ovos \\
\hline \multicolumn{4}{|c|}{ Juvenis $100 \mathrm{~cm}^{3}$} \\
\hline Amen. 3 & 100,0 & 20,0 & 300,0 \\
\hline Amen. 6 & 700,0 & 60,5 & 800,0 \\
\hline Cana 3 & 440,0 & 20,5 & 320,0 \\
\hline Cana 6 & 800,0 & 120,0 & 840,0 \\
\hline $\mathrm{T}$ & 860,0 & 140,0 & 880,0 \\
\hline \multicolumn{4}{|c|}{ * Difere da testemunha pelo teste de Dunnet a 5\% de probabilidade. } \\
\hline Fatorial & Meloidogyne & Pratylenchus & ovos \\
\hline Resíduos & \multicolumn{2}{|c|}{ Juvenis $100 \mathrm{~cm}^{3}$} & \\
\hline Amen. & 400,0 a & 40,0 a & $550,0 \mathrm{a}$ \\
\hline Cana & $620,0 \mathrm{a}$ & 70,0 a & $580,0 \mathrm{a}$ \\
\hline Níveis & & & \\
\hline $3 \mathrm{~cm}$ & $270,0 \mathrm{~b}$ & 20,0 a & $310,0 \mathrm{~b}$ \\
\hline $6 \mathrm{~cm}$ & 750,0 a & 90,0 a & $820,0 \mathrm{a}$ \\
\hline
\end{tabular}

Letras iguais nas colunas não diferem significativamente pelo teste de Tukey a $5 \%$ de probabilidade.

No segundo ciclo produtivo da alface não foi verificada a presença de nematoides do gênero Pratylenchus. A população de Meloidogyne diferiu do tratamento controle apenas no resíduo com $3 \mathrm{~cm}$ de cana de açúcar apresentando maior número desses nematoides. No número de ovos os tratamentos não diferiram do tratamento controle (Tabela 2).

Não houveram diferenças quando foram comparadas as duas espécies de resíduos utilizados, mas para os níveis de cobertura a camada de $3 \mathrm{~cm}$ apresentou maior população de nematoides do gênero Meloidogyne (Tabela 2). 
Tabela 2 - População de nematoides no solo na produção no segundo cultivo de alface sobre diferentes resíduos, em diferentes alturas.

\begin{tabular}{|c|c|c|}
\hline Fatorial $\mathrm{x}$ testemunha & Meloidogyne & ovos \\
\hline \multicolumn{3}{|c|}{ Juvenis $100 \mathrm{~cm}^{3}$} \\
\hline Amen. 3 & 160,0 & 140,0 \\
\hline Amen. 6 & 160,0 & 260,0 \\
\hline Cana 3 & $240,0^{*}$ & 380,0 \\
\hline Cana 6 & 20,0 & 240,0 \\
\hline $\mathrm{T}$ & 120,0 & 300,0 \\
\hline \multicolumn{3}{|c|}{ * Difere da testemunha pelo teste de Dunnet a 5\% de probabilidade. } \\
\hline Fatorial & Meloidogyne & ovos \\
\hline Resíduos & Juvenis $100 \mathrm{~cm}^{3}$ & \\
\hline Amen. & 160,0 a & 200,0 a \\
\hline Cana & 130,0 a & 310,0 a \\
\hline \multicolumn{3}{|l|}{ Níveis } \\
\hline $3 \mathrm{~cm}$ & 200,0 a & $260,0 \mathrm{a}$ \\
\hline $6 \mathrm{~cm}$ & $90,0 \mathrm{~b}$ & $250,0 \mathrm{a}$ \\
\hline
\end{tabular}

Letras iguais nas colunas não diferem significativamente pelo teste de Tukey a $5 \%$ de probabilidade.

Os tratamentos influenciaram entre si na população de Meloidogyne, a utilização da camada de $3 \mathrm{~cm}$ de cana-de-açúcar aumentou em 12 vezes em relação ao mesmo resíduo com 6 $\mathrm{cm}$, e em 8 vezes quando comparado ao resíduo de $6 \mathrm{~cm}$ de amendoim (Tabela 3).

Tabela 3 - Número de nematoides do gênero Meloidogyne, no solo, na camada de 0 a $20 \mathrm{~cm}$, decorrentes da interação entre resíduos e níveis de cobertura sob o solo na produção do segundo ciclo produtivo da alface.

\begin{tabular}{lcc} 
& \multicolumn{3}{c}{ Juvenis $100 \mathrm{~cm}^{3}$} \\
\cline { 2 - 4 } & $3 \mathrm{~cm}$ & $6 \mathrm{~cm}$ \\
\hline Amendoim & $160,0 \mathrm{aA}$ & $160,0 \mathrm{aA}$ \\
Cana & $240,0 \mathrm{aA}$ & $20,0 \mathrm{bB}$ \\
\hline CV(\%) & & 37,62 \\
\hline
\end{tabular}

Médias seguidas de mesma letra, maiúscula nas linhas, minúscula nas colunas, não diferem estatisticamente pelo teste Tukey (5\%).

\section{DISCUSSÃO}

Neste trabalho, observamos que a população de nematoides do gênero Meloidogyne obteve um aumento no primeiro ciclo de cultivo da cultura da alface, quando a espessura da camada de cobertura morta sobre o solo foi de seis centímetros de altura. Segundo Rinaldi et al. (2014), a constituição de espécies de nematoides é consequência do substrato, do clima, dos elementos orgânicos e disfunções naturais e antrópicas. Isto é, certas mudanças nas práticas do solo e na cobertura altera a oferta de seus recursos e reflete na diversidade da comunidade de nematoides (RITZINGER et al., 2011).

Há situações em que a cobertura morta colabora para o surgimento de pragas e doenças no solo. Isso acontece quando se utiliza restos culturais já contaminados ou então, quando o 
aumento da umidade, consequência da cobertura morta, propicia o desenvolvimento destes (FONSECA; ANGELETTI, 1987). Solos com temperaturas em torno de 25 a $30^{\circ} \mathrm{C}$ são considerados ideais para o desenvolvimento de $M$. arenaria, $M$. incognita e $M$. javanica (FERRAZ; MONTEIRO, 1995) e teor de argila inferior a 20\%, favorecem de 10 à 20 vezes a proliferação de Meidogyne javanica (NEMATOLOGIA, 2012).

A camada de resíduos orgânicos sobre o solo, exerce significativa influência na temperatura da área. Essa interferência varia de acordo com o tipo e a espessura do material orgânico usado. Todos os tipos de materiais como, cascas de arroz, de café entre outras, reduzem a amplitude térmica diária do solo (FONSECA; ANGELETTI, 1987). Como vimos, essas afirmações científicas possibilitam fundamentar o resultado apresentado nesta pesquisa.

Oliveira et al. (2009), concluíram que compostos orgânicos sobre o solo enriquecem a biomassa microbiana em virtude das elevadas proporções de carbono e nitrogênio lábeis motivando diretamente a microbiota do solo. Portanto, a conservação da cobertura do solo e o uso de resíduos orgânicos, contribuem para a atividade dos microrganismos.

Em relação ao segundo ciclo de cultivo da alface, não houve presença de nematoide do gênero Pratylenchus, enquanto que a população do gênero Meloidogyne aumentou. De acordo com Walker (1989), quanto maior a densidade populacional de uma fauna em uma cobertura vegetal, maior será a probabilidade desta comunidade estar predominando, e consequentemente, restringindo o equilíbrio do meio, já que, a variabilidade de espécies encontra-se relacionada a um vínculo entre número de espécies (riqueza de espécies) e a disseminação do número de indivíduos entre as estirpes (equitabilidade).

Em um trabalho feito por Oliveira et al. (2006) sobre os efeitos da adição de diferentes resíduos culturais ao solo sobre a população do nematoide de cisto da soja, os tratamentos que utilizaram a palhada de Crotalaria juncea e o bagaço de cana, o número de fêmeas encontradas nas raízes foi maior do que nas plantas controle. Em nossa pesquisa, o número de nematoide Meloidogyne foi maior no resíduo de bagaço de cana do que no resíduo de casca de amendoim.

\section{CONCLUSÃO}

A cobertura morta composta pelo bagaço de cana-de-açúcar ganhou destaque referente ao aumento populacional de nematoides do gênero Meloidogyne. A camada com espessura de $3 \mathrm{~cm}$ de resíduo de cana-de-açúcar foi suficiente para elevar a população de nematoides no solo.

\section{REFERÊNCIAS}

AITA C; GIACOMINI SJ. Decomposição e liberação de nitrogênio de resíduos culturais de plantas de cobertura de solo solteiras e consorciadas. Revista Brasileira de Ciência do Solo. 27: 601-612, 2003. https://doi.org/10.1590/S0100-06832003000400004

ALENCAR, T. A. et al. Efeito de intervalos de aplicação de urina bovina na produção de alface em cultivo protegido. Revista Verde. Mossoró, v.7, n.3, p. 53-67, 2012.

ARIEIRA, C. R. D.; FERRAZ, S.; FREITAS, L.G.; MIZOBUTSI, E. H. Avaliação de gramíneas forrageiras para o controle de Meloidogyne incognita e M. javanica (Nematoda). Acta Scientiarum. Agronomy Maringá, v. 25, no. 2, p. 473-477, 2003. Disponível em: < 
https://docs.google.com/viewerng/viewer?url=http://ojs.uem.br/ojs/index.php/ActaSciAgron/arti cle/viewFile/2163/1614> Acesso em 29 jul 2017.

DIAS, P. S. Desempenho dos carvões obtidos a partir das biomassas caroço de piquí e da casca do cupuaçú em processos de adsorção de azul de metileno em meio aquoso. 2013 43p. Dissertação (Mestrado) - Universidade Estadual do Sudoeste da Bahia. Itapetinga, BA.

ESPINDOLA, J. A. A. et al. Decomposição e liberação de nutrientes acumulados em leguminosas herbáceas perenes consorciadas com bananeira. Revista Brasileira de Ciência do Solo. 30: 321328, 2006. https://doi.org/10.1590/S0100-06832006000200012

FONSECA, A. F. A.; ANGELETTI, M. P. Utilização de cobertura morta do solo para a produção de hortaliças no Estado de Rondônia. Porto Velho, EMBRAPA - UEPAE. Porto Velho, 1987.

FERRAZ, L. C. C. B.; MONTEIRO, A. R. Nematóides. In: BERGAMIM FILHO, A.; KIMATI, H.; AMORIN, L. Manual de Fitopatologia: princípios e conceitos, v.1, 3 ed, São Paulo: Ceres, 1995. p. 168-201.

JENKINS WR. 1964. A rapid centrifugal- flotation technique for separating nematodes from soil. Plant Disease Report 48: 692.

LIMA, T. M. et al. Análise qualitativa das cascas de amendoim: uma alternativa de uso como fertilizantes orgânico. Cadernos de Agroecologia, vol. 5 N. 1. MS: UFG, 2010. 2 p.

MORAES, S. R. G.;CAMPOS, V. P.; POZZA, E. A.; FONTANETTI, A.; CARVALHO, G. J.; MAXIMINIANO, C. Influência de Leguminosas no Controle de Fitonematóides no Cultivo Orgânico de Alface Americana e de Repolho. Fitopatologia Brasileira. 2006. Disponível em: < http://www.scielo.br/pdf/\%0D/fb/v31n2/30014.pdf> Acesso em : 29 jul 2017.

NEMATOLOGIA. Culturas de cobertura e de rotação devem ser plantas não hospedeiras de nematoides. 2012. Disponível em: http://nematologia.com.br/wpcontent/uploads/2012/07/guima9.pdf Acesso em: 5 jul. 2017

OLIVEIRA, L. C.; STANGARLIN, J. R.; LANA, M. do C., SIMON, D.; ZIMMERMANN, A. Biomassa microbiana em cultivo de alface sob diferentes adubações orgânicas e manejo da adubação verde. Revista Brasileira de Agroecologia, v.4, p.182-185, 2009.

OLIVEIRA, F. S.; ROCHA, M. R.; COSTA, R. B.; MACHADO, V. O. F.; NOGUEIRA, E. N. Efeito da adição de diferentes resíduos culturais ao solo sob a população de nematóide de cisto da soja.

Nematologia Brasileira, v. 30, n. 2, p. 171-177, 2006.

PADOVAN, M. P. et al. Avaliação de cultivares de soja, sob manejo orgânico, para fins de adubação verde e produção de grãos. Pesquisa Agropecuária Brasileira. 37: 1705-1710, 2002. https://doi.org/10.1590/S0100-204X2002001200005 
RITZINGER, C. H. S.P.; FANCELLI, M. MANEJO INTEGRADO DE NEMATÓIDES NA CULTURA DA

BANANEIRA. Rev. Bras. Frutic., Jaboticabal - SP, v. 28, n. 2, p. 331-338, Agosto 2006.

SANTOS, C. A. P. dos. Produção da alface crespa e umidade do solo em função de diferentes fontes de matéria orgânica e cobertura do solo. 2011. 52 p. Dissertação (Mestrado). Universidade Federal do Sergipe, São Cristóvão.

WALKER, D. Diversity and stability. In: CHERRETT, J.M., ed. Ecological concepts. Oxford, Blackwell Scientific Public, 1989. p. 115-146.

WASILEWSKA, L. Impact of human activities on nematode communities in terrestrial ecosystems. In: Ecology of Arable Land-Perspectives and Challenges. Springer Netherlands, 1989. p. 123-132. https://doi.org/10.1007/978-94-009-1021-8 12

YEATES, G. W. Variation in soil nematode diversity under pasture with soil and year.

Soil Biology and Biochemistry, v. 16, n. 2, p. 95-102. 1984. https://doi.org/10.1016/0038$\underline{0717(84) 90098-1}$ 